\title{
Coupled Quantum Dots as Two-Level Systems: A Variational Monte Carlo Approach
}

\author{
J. Kim, D.V. Melnikov and J-.P. Leburton
}

Beckman Institute for Advanced Science \& Technology and Department of Electrical and Computer Engineering,
University of Illinois at Urbana-Champaign, 405 N. Mathews Avenue, Urbana, Illinois 61801, USA

\begin{abstract}
The electronic properties of two-dimensional double-quantum dots in the presence of external magnetic fields are investigated by a variational Monte Carlo method with $s$ and $s-p$ trial wavefunctions. We compute the exchange energy between two electrons as well as the two-electron total Coulomb energy for the singlet and triplet states in both strongly and weakly coupled quantum dots, and compare our data with the results of the numerically exact diagonalization of the Schrödinger Equation. In both systems, the singlet Coulomb energy decreases in magnetic fields as a consequence of magnetic localization, whereas the triplet Coulomb energy reaches a maximum value at intermediate magnetic fields before decreasing. Overall, good agreement between the two methods is obtained with $s-p$ orbital trial wavefunction in strongly and weakly coupled quantum dots.
\end{abstract}

\section{INTRODUCTION}

Recent works indicate that coupled quantum dots (QD) are promising nanostructures for realizing quantum gates that are the basic elements of a quantum computer $[1,2]$. In QDs, fundamental quantum logic operations (e.g. controlNOT operation) can be achieved in a quantum gate by controlling the entanglement between two electron spins by means of external electric and magnetic fields [3]. The entanglement between the spins or qubits can be quantified by calculating the exchange energy $J$, which is the energy difference between the lowest triplet and the singlet states [1]. Proper operation of a quantum gate largely depends on the ability to control and to modulate the exchange energy with external voltages and magnetic fields. Recently, the coherent manipulation of the two-electron spin state in coupled quantum dots has been demonstrated, which is an important milestone toward the realization of a quantum gate with solidstate systems [4]. However, measuring and controlling the exchange energy between two spins still remains a formidable task in experimental coupled QDs. In this context numerical simulations provide a valuable tool to gain insights into the complexity of the basic physical effects at play in the QD and their manipulation in a device environment.

Among various theoretical modeling approaches, quantum Monte Carlo techniques offer simple yet accurate ways to simulate many-particle quantum systems $[5,6]$. In particular, variational Monte Carlo method (VMC) relies on a set of trial wavefunctions with adjustable parameters to derive by variational technique the total energy of the system; the latter being estimated by random number generation. The benefits of using VMC include (1) relatively simple implementation, (2) low memory requirement in the numerical method, and

*Address correspondence to this author at the Beckman Institute for Ad-
vanced Science \& Technology and Department of Electrical and Computer
Engineering, University of Illinois at Urbana-Champaign, 405 N., Mathews Engineering, University of Illinois at Urbana-Champaign, 405 N.,
Avenue, Urbana, Illinois 61801, USA; E-mail: jihankim@uiuc.edu
(3) easy parallelization due to the inherent parallel nature of Monte Carlo. In our work, we use the variational Monte Carlo method to compute the exchange and Coulomb energies of two electrons in coupled QD systems in presence of magnetic fields, thereby extending the previous works on single QDs [7, 8] and double QDs in zero magnetic fields [9]. For comparison purposes, we also include results obtained from numerical diagonalization of the many-body Schrödinger Equation (exact diagonalization or ED method) [10].

The paper is divided into four sections. In Sec. II, we describe the computational approach used to solve the manybody Schrödinger Equation. In Sec. III, we provide results of our simulations for both the strongly coupled and weakly coupled QDs and analyze the important differences between the two cases. Finally, in Sec. IV, we summarize the important points mentioned in the paper.

\section{MATERIALS AND METHODOLOGY}

The general model Hamiltonian for $\mathrm{N}$ electrons in coupled QDs can be written as follows

$$
\mathrm{H}=\sum_{j=1}^{N}\left\{\frac{\left(-i \hbar \vec{\nabla}_{j}-\frac{e}{c} \vec{A}_{j}\right)^{2}}{2 m^{*}}+V_{e x t}\left(\vec{r}_{j}\right)\right\}+\sum_{j<k}^{N} \frac{e^{2}}{\varepsilon\left|\vec{r}_{j}-\vec{r}_{k}\right|}
$$

where we use the effective-mass approximation to describe the many-body effects among the electrons in the conduction band in the two-dimensional (2D) $x y$-plane, and neglect the extension of the wavefunction along the $\mathrm{z}$-direction in a first approximation [11]. Here

$$
\mathrm{V}_{\mathrm{ext}}\left(x_{i}, y_{i}\right)=\frac{1}{2} m * \omega_{0}^{2} \min \left[\left(x_{i}-a\right)^{2}+y_{i}^{2},\left(x_{i}+a\right)^{2}+y_{i}^{2}\right]
$$

is the $2 \mathrm{D}$ external potential of the double QD, which we model as a double parabola with two minima located at $x= \pm$ $a$ along the $x$-axis for the sake of simplicity. The radial fre- 
quency $\omega_{0}$ indicates the confinement strength, which we set $\hbar \omega_{0}=3 \mathrm{meV}$ to be consistent with experimental data for gated QDs [12]. The GaAs effective mass is set to be $m^{*}=0.067 m_{0}$, with $m_{0}$ being the free electron mass, and the dielectric constant is $\varepsilon=12.7 . \vec{A}$ is the vector potential experienced by each electron, which is expressed as

$\vec{A}=\frac{B}{2}(y,-x)$

in the $2 \mathrm{D}$ symmetric gauge, where $B$ is the magnetic field oriented along the $z$-direction. We ignore Zeeman splitting in the current analysis.

In VMC the most commonly used trial wavefunction has a Slater-Jastrow form [13], which is a product of Slater determinants consisting of single-particle orbitals for spin up and spin down electrons ( $D^{\uparrow}$ and $D^{\downarrow}$ respectively) combined with a Jastrow term, $\mathfrak{J}\left(r_{i j}\right)$ to account for electron correlations, i.e.

$\Psi_{\mathrm{T}}=D^{\uparrow} D^{\downarrow} \prod_{i<j}^{N} \mathfrak{I}\left(r_{i j}\right)$,

where $r_{i j}=\left|\vec{r}_{i}-\vec{r}_{j}\right|$.

In our simulations, we focus on the special case $\mathrm{N}=2$ electrons and set the two-body Jastrow term to be

$\mathfrak{I}\left(r_{12}\right)=e^{\frac{a_{1} r_{12}}{1+b_{1} r_{12}}}$

where $a_{1}$ is fixed by the "cusp" condition to be 0.5 for singlet and 0.25 for triplet [14]. We neglect three-body and higher correlation terms in the Jastrow term. We replace the Slater determinants with the following expressions for the lowest singlet and the triplet trial wavefunctions of two electrons, respectively.

$$
\begin{aligned}
& \left(D^{\uparrow} D^{\downarrow}\right)_{\mathrm{S}}=\sum_{i, j} \alpha_{i j}\left(\phi_{i}\left(r_{1}\right) \phi_{j}\left(r_{2}\right)+\phi_{j}\left(r_{1}\right) \phi_{i}\left(r_{2}\right)\right) \\
& \left(D^{\uparrow} D^{\downarrow}\right)_{\mathrm{T}}=\sum_{i, j} \alpha_{i j}\left(\phi_{i}\left(r_{1}\right) \phi_{j}\left(r_{2}\right)-\phi_{j}\left(r_{1}\right) \phi_{i}\left(r_{2}\right)\right)
\end{aligned}
$$

Here, we use the following expressions for single particle $s$ orbitals

$\phi_{j}\left(\vec{r}_{k}\right)=e^{-\alpha_{x}^{2}\left[x_{k}+(-1)^{j-1} \frac{d}{2}\right]^{2} / 2} e^{-\alpha_{y}^{2} y_{k}^{2} / 2} e^{i(-1)^{j} \alpha_{b} y_{k}} \mathrm{j}=1,2$

and for single particle $p$ orbitals

$$
\phi_{j}\left(\vec{r}_{k}\right)=\left[\left(x_{k}+d_{2}\right)+i(-1)^{j-1} y_{k}\right] e^{-\alpha_{x}^{2}\left[x_{k}+\frac{d}{2}\right]^{2} / 2} e^{-\alpha_{y}^{2} y_{k}^{2} / 2} e^{i(-1)^{j} \alpha_{b} y_{k}}
$$$$
\mathrm{j}=3,4
$$

$\phi_{j}\left(\vec{r}_{k}\right)=\left[\left(x_{k}-d_{2}\right)+i(-1)^{j-1} y_{k}\right] e^{-\alpha_{x}^{2}\left[x_{k}-\frac{d}{2}\right]^{2} / 2} e^{-\alpha_{y}^{2} y_{k}^{2} / 2} e^{i(-1)^{j} \alpha_{b} y_{k}}$

$\mathrm{j}=5,6$ where $\alpha_{\mathrm{ij}}, \alpha_{\mathrm{x}}, \alpha_{\mathrm{y}}, \mathrm{d}, \mathrm{d}_{2}$, and $\alpha_{\mathrm{b}}$ are treated as variational parameters, and $r_{k}=\left(x_{k}, y_{k}\right)$. The single particle orbitals (Eq. (7) and Eq. (8)), are the two lowest states of circular single dot eigenstates (Fock-Darwin states) localized in the left and right quantum dots. For some sets of simulations, we only vary $\alpha_{i j}$, the coefficients in front of the single particle orbitals, while keeping other variational parameters fixed [15].

$$
\begin{aligned}
& \alpha_{x}=\alpha_{y}=\frac{\sqrt[4]{q^{2} B^{2} / 4+\omega_{o}^{2} m^{* 2} c^{2}}}{\sqrt{\hbar c}}, \\
& d=d_{2}=a, \alpha_{b}=\frac{a q^{2} B^{2}}{2 \hbar c} .
\end{aligned}
$$

For the singlet wavefunction with just the $s$ orbitals (Eq. (5) and Eq. (7)), $\alpha_{11}$ and $\alpha_{22}$ are the variational parameters that determine the electron double occupation probability (situation in which two electrons occupy the same quantum dot) [16]. It is also seen that when $\mathfrak{I}\left(r_{i j}\right)=1$, double occupation is zero at $\alpha_{11}=\alpha_{22}=0$ as the wavefunction represents a Heitler-London state. The VMC triplet wavefunction for $s$ orbitals (Eq. (6) and Eq. (7)) formally coincides with HundMulliken wavefunction when $\mathfrak{I}\left(r_{i j}\right)=1$ [16].

To numerically optimize the expectation value of the energy, we use the Steepest Descent method [17] in which variational parameters $\alpha_{k}^{\prime}$ are updated as $\alpha_{k} \rightarrow \alpha_{k}+\Delta t f_{k}$ at each iteration, where

$$
\begin{aligned}
& f_{k}=-\frac{\partial E}{\partial \alpha_{k}}=-\frac{\left\langle\Psi\left|O_{k}^{*} H+H O_{k}+\partial_{\alpha_{k}} H\right| \Psi\right\rangle}{\langle\Psi \mid \Psi\rangle}+ \\
& \frac{\left\langle\Psi\left|O_{k}\right| \Psi\right\rangle\langle\Psi|H| \Psi\rangle}{\langle\Psi \mid \Psi\rangle}+\frac{\left\langle\Psi\left|O_{k}^{*}\right| \Psi\right\rangle\left\langle\Psi\left|O_{k}\right| \Psi\right\rangle}{\langle\Psi|\Psi|\rangle}
\end{aligned}
$$

with $\Psi$ being the trial wavefunction,

$$
O_{k}=\frac{\partial}{\partial \alpha_{k}} \ln \Psi \text {, and } O_{k}^{*}=\frac{\partial}{\partial \alpha_{k}} \ln \Psi^{*} \text {, and } \mathrm{E}=\frac{\langle\Psi|H| \Psi\rangle}{\langle\Psi \mid \Psi\rangle}
$$

is the total energy. $\Delta t$ is chosen such that after an adequate number of changes in the sign of $f_{k}$, it becomes inversely proportional to the total number of iterations, allowing the variational parameters to converge numerically. By using a sufficiently large number of random walkers $N_{w}$, we solve for the expectation values of all the observables in Eq. (10) by utilizing the Monte Carlo integration

$$
\mathrm{E}=\frac{\langle\Psi|H| \Psi\rangle}{\langle\Psi \mid \Psi\rangle} \approx \frac{1}{\mathrm{~N}_{\mathrm{w}}} \sum E_{L}\left(R_{i}\right)
$$

where $E_{L}$ is the local energy and $R_{i}$ is ith electron positions from the probability distribution, which is derived from the Metropolis algorithm.

\section{RESULTS}

We calculated the total energy of the $\mathrm{N}=2$ electrons system for both the singlet $\left(E_{\mathrm{S}}\right)$ and the triplet $\left(E_{\mathrm{T}}\right)$ states in the coupled QD with nine different approaches: (1) by using the ED method described in Ref. [11], (2) by using VMC with 
the Jastrow term and $s$ orbitals, (3) by using VMC with $s$ orbitals but setting the Jastrow term $\mathfrak{I}=1$, (4) by using VMC with the Jastrow term and $s-p$ orbitals, (5) by using VMC with $s-p$ orbitals with the Jastrow term $\mathfrak{I}=1$, (6) by using $\mathrm{VMC}$ with the Jastrow term, $s$ orbitals, and fixed variational parameters (Eq. 9), (7) by using VMC with $\mathfrak{I}=1, s$ orbitals, and fixed variational parameters, (8) by using VMC with the Jastrow term, $s-p$ orbitals, and fixed variational parameters, and (9) by using VMC with $s-p$ orbitals with $\mathfrak{I}=1$, and fixed variational parameters. In Fig. (1a), we display the ED results, $J=E_{\mathrm{T}}-E_{\mathrm{S}}$, at various magnetic fields, for which the separation between the two potential minima in Eq. (3) along the $x$-axis is $2 a=30 \mathrm{~nm}$ (strongly coupled case). In Fig. (1b), we plot the difference in total energies obtained from various VMC trial wavefunctions from the ED results from Fig. (1a) to compare the various different VMC approaches. Comparing all VMC results with those obtained by ED method, we can see that the presence of the Jastrow term improves the accuracy of the VMC technique as expected. In Fig. (1b) for $\mathrm{B}=0 \mathrm{~T}$, the accuracy improves from 202.4 to $17.6 \mu \mathrm{eV}$, from -12.23 to $-2.49 \mu \mathrm{eV}$, from 747.1 to $86.05 \mu \mathrm{eV}$, and from 110.5 to $41.88 \mu \mathrm{eV}$ for VMC using $s$ orbitals, $s-p$ orbitals, fixed variational $s$ orbitals, and fixed variational $s-p$ orbitals, respectively. However, at higher magnetic field values, the improvement is less pronounced or even negligible depending on the specific regime and the specific trial wavefunctions. This can be easily explained by noting that since $\mathfrak{I}$ accounts for correlation effects between electrons, we expect it to have a weaker effect at high magnetic fields where the two electrons are more decoupled.

In Fig. (1b), we see that the two s orbital curves look similar to one another from $\mathrm{B}=2$ to $6 \mathrm{~T}$, which is expected since the effect of $\mathfrak{I}$ diminishes with higher magnetic fields as mentioned earlier. We see that in both curves, the error increases when $\mathrm{B}>2 \mathrm{~T}$ with a peak value occurring at $3.5 \mathrm{~T}$ (73.9 $\mu \mathrm{eV}$ for $\mathrm{s}$ orbital without Jastrow term and $61.8 \mu \mathrm{eV}$ for s orbital with Jastrow). It turns out that at magnetic fields around 3 to $5 \mathrm{~T}$, higher energy single-particle orbitals contribute significantly to the singlet two-electron wavefunctions [11]. Because such states are omitted in the $s$ orbital VMC trial wavefunctions, deviations from the actual results are accentuated at this field range. Unlike the singlet state in this interval of magnetic fields, the true triplet state is actually relatively well described by wavefunction that does not include other high energy orbitals. Overall, the two effects result in lower than expected VMC exchange energies over this magnetic field range. Upon including the $p$ orbitals, we

see the accuracy of the VMC results improves significantly at this magnetic field range. We also note that even with the inclusion of $p$ orbitals, the Jastrow term does play a role in improving accuracy at low magnetic fields $(\mathrm{B}=0$ to $2 \mathrm{~T}$ ). Finally, trial wavefunctions with fixed variational parameters give inaccurate results, showing the importance of optimizing these parameters in strongly coupled QDs. (a)

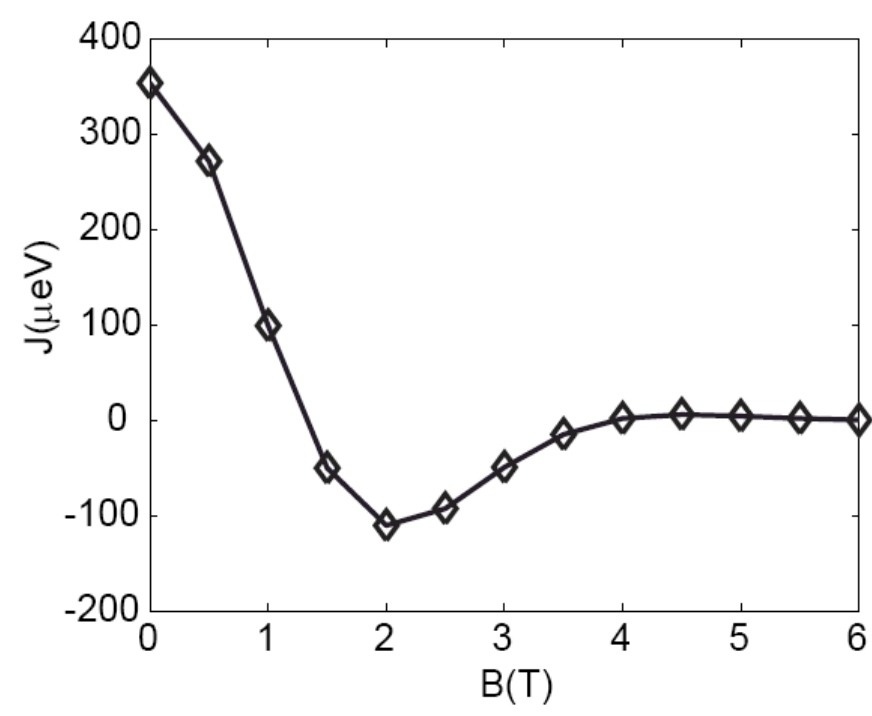

(b)
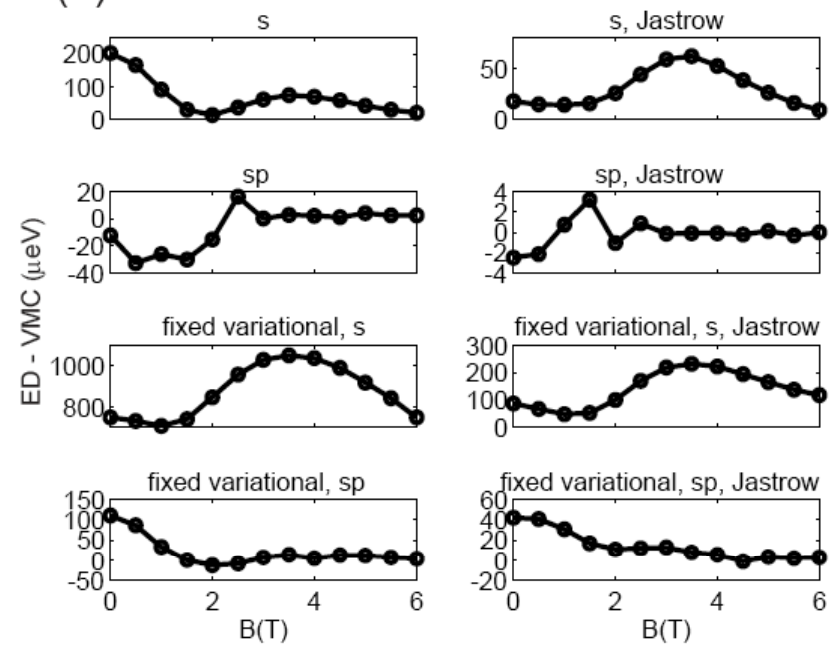

Fig. (1). Simulated exchange energy versus magnetic fields in strongly coupled QDs (potential minima separated by $30 \mathrm{~nm}$ ). (a) ED method and (b) difference between the energies obtained from ED and the different VMC trial wavefunctions.

In Fig. (2a,b), we plot the total Coulomb energy (TCE)

$$
\left\langle\Psi\left|\frac{e^{2}}{\varepsilon r_{12}}\right| \Psi\right\rangle
$$

between the two electrons for the singlet and triplet states. Both the direct Coulomb interaction and the exchange interaction contribute to the TCE, so its behavior depends on the interplay between these two terms: in the singlet state, both are positive, while in the triplet state, the exchange interaction is negative. We observe that the singlet TCE given by $s$ orbital VMC calculations decreases monotonically with increasing magnetic fields, while the ED and the $s-p$ orbital VMC results indicate a minimum at around $3 \mathrm{~T}$ (Fig. 2a). Physically, at small magnetic fields, the singlet TCE decreases as the electrons gradually localize in each QD, and the overlap between them diminishes. However, in strongly coupled QDs, higher 
(a)
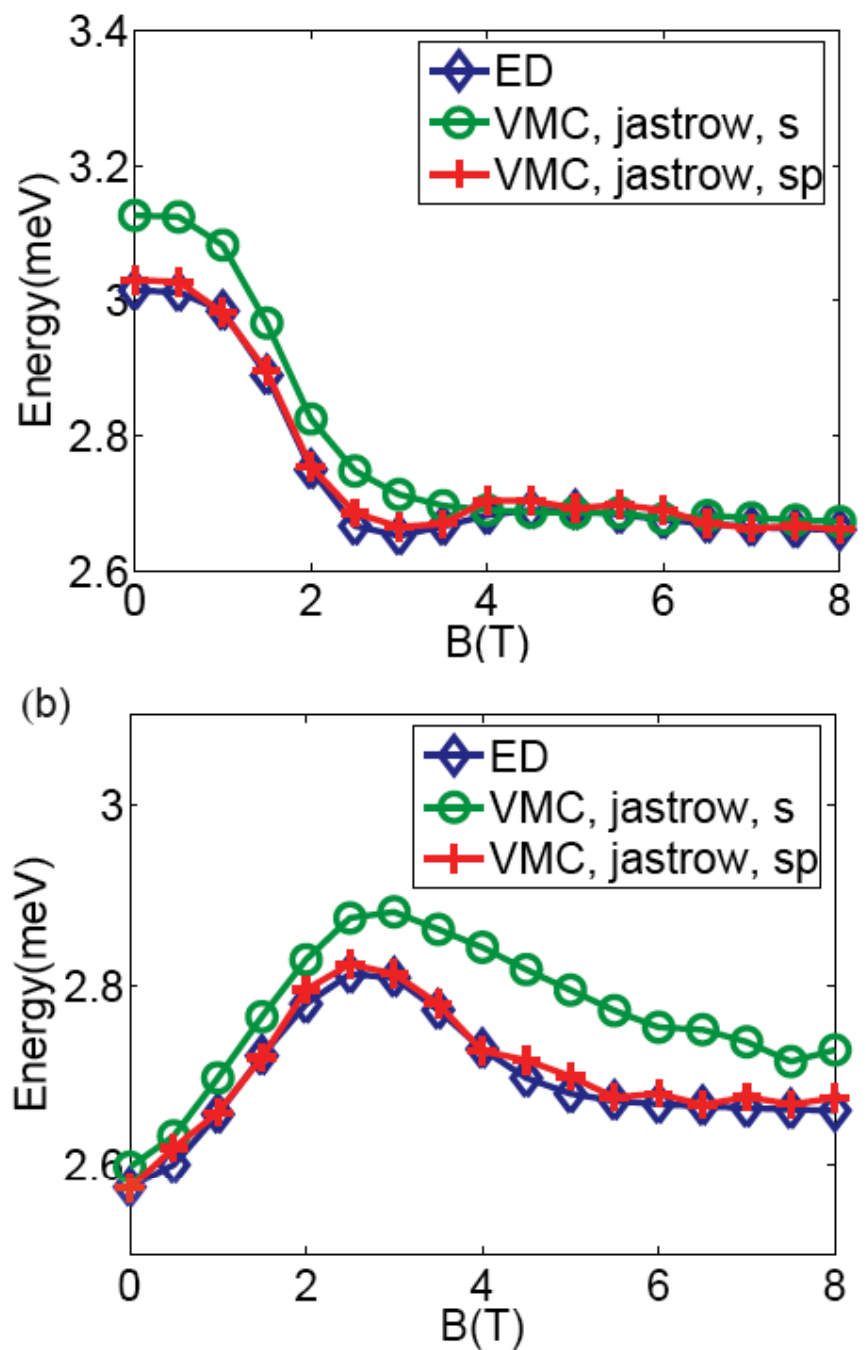

Fig. (2). Total Coulomb energy vs magnetic field in strongly coupled QDs. (a) singlet state and (b) triplet state. 'o'-symbols: VMC ( $s$ orbital); '+'-symbols: VMC ( $s-p$ orbital); ' $\diamond$ '- symbols: ED calculations.

energy single-particle orbitals (neglected in $s$ orbital VMC) contribute to the many-body state as the system strives to decrease its total energy (at around $\sim 3 \mathrm{~T}$ in our simulation) [11] as electrons effectively become more localized. In this case, even though the orbital energy increases with magnetic field, the TCE decreases (this effect is reminiscent of the energy behavior of a two-electron pair in single circular QDs [11]). Hence, at low magnetic fields, the ED and the $s-p$ orbital Coulomb expectation values decrease faster than the VMC values calculated with only the two lowest single-particle orbitals. As the magnetic field increases further, the Coulomb expectation values approach to the same values in all three cases as the increasing magnetic field just localizes electrons in separate QDs in a configuration similar to Heitler-London state. In the triplet state, the TCE calculated by VMC and ED, exhibits a maximum around $\mathrm{B}=3 \mathrm{~T}$, and decreases afterward (Fig. 2b). Unlike the singlet state, this behavior arises from the difference between the direct repulsion between electrons and the exchange interaction in the TCE. Hence, while both terms decrease with magnetic fields the faster decay of the latter results in the TCE rise at low magnetic fields [18].

In Fig. (3a), we plot the ED exchange energy for a weakly coupled quantum dot system, for which we set the separation between the two potential minima to be $60 \mathrm{~nm}$, while keeping $\omega_{0}$ at $3 \mathrm{meV}$. Because the confining potential is quadratic in $\omega_{0}$ (Eq. 2) an alternative way to decouple the QDs is to increase the confinement strength. Similarly to the strongly coupled QD case, we plot the difference in total energies obtained from various VMC trial wavefunctions

(a)

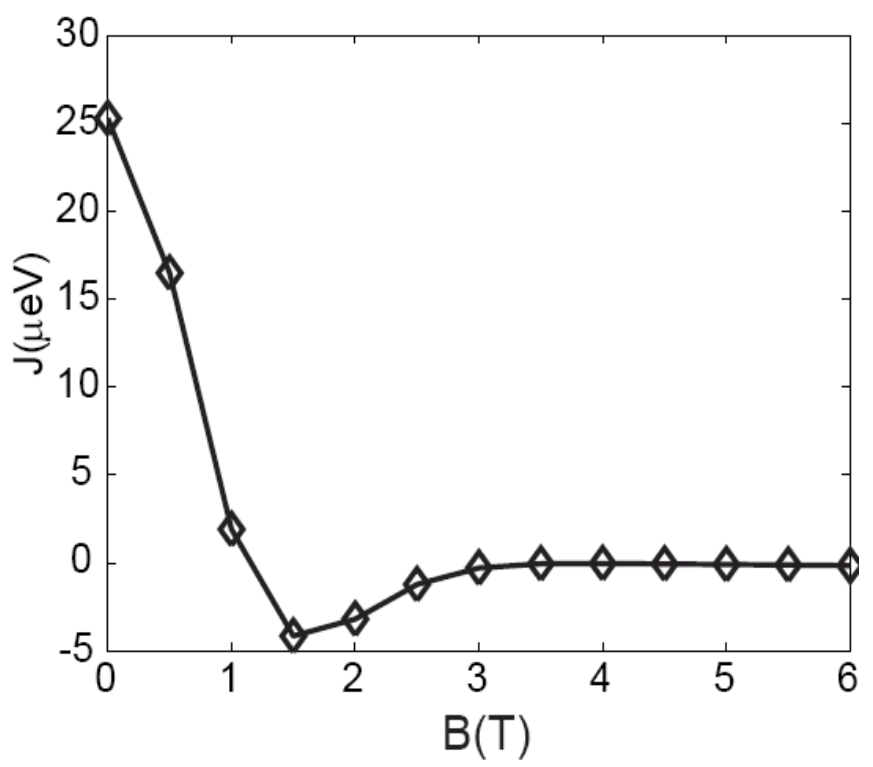

(b)
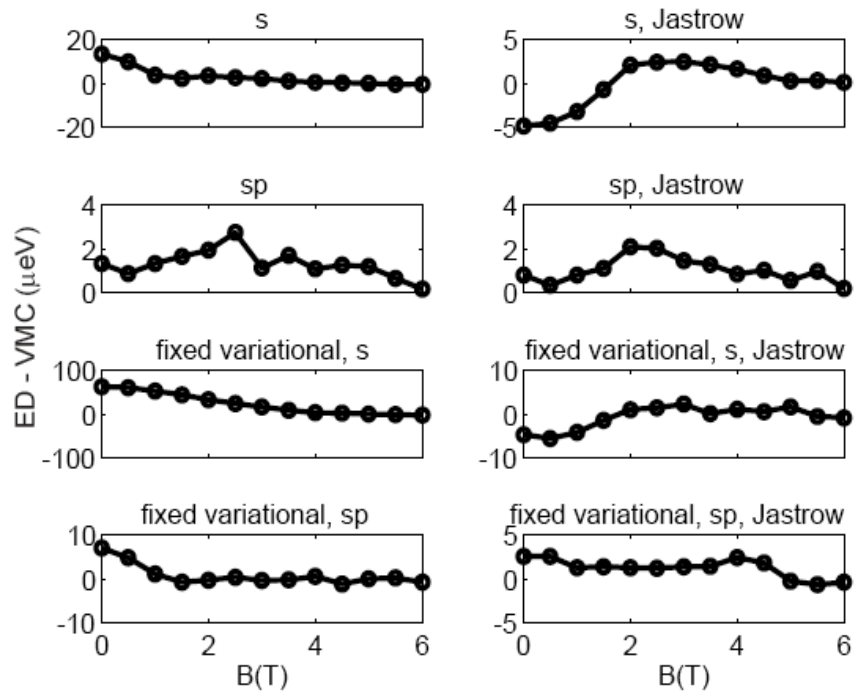

Fig. (3). Same as in Fig. (1a,b) but for the weakly coupled QDs (potential minima separated by $60 \mathrm{~nm}$ ).

from the $\mathrm{ED}$ results in Fig. (3b). In Fig. (3b) at $\mathrm{B}=0 \mathrm{~T}$, we notice the exchange energies are about one order of magnitude smaller than in strongly coupled QDs (Fig. 1), which is expected since the wavefunction overlap between the two electrons strongly decreases with the dot separation. Com- 
paring Figs. (1b) and (3b), we see closer agreement between all of the VMC and the ED results in weakly coupled dots with noticeable improvements in the $s$ orbital VMC wavefunction cases. This can be explained by the fact that upon increasing the distance between the QDs, the contribution of the high energy single-particle orbitals to the singlet wavefunctions, which are omitted in the $s$ orbital VMC simulations, becomes negligible. Also, we observe the weaker role of the $\mathfrak{I}$ in the weakly coupled dots. In fact, there isn't any statistically significant difference in accuracy between the two $s-p$ orbital cases (one with Jastrow term and the other without) since correlation between electrons, which is accounted for by the Jastrow term, becomes less important as the dots become more decoupled.

Finally, in Fig. (4a), we observe a general behavior of the evolution of the singlet Coulomb energies different from the strongly coupled QDs. Specifically, the local minimum in the TCE, which was found in strongly coupled QDs with the ED calculations, disappears thereby reconciling both the $s$ orbital VMC and the ED approaches. Also, from $\mathrm{B}=0$ to $\mathrm{B}$ $=4 \mathrm{~T}$, the total reduction in the singlet TCE is $\sim 0.08 \mathrm{meV}$, a value much smaller than $\sim 0.5 \mathrm{meV}$ calculated in strongly coupled QDs, which again reflects the decoupled nature of the electrons in the weakly coupled QDs. In the triplet state (Fig. 4b), the TCE calculated by VMC and ED, exhibits a maximum around $\mathrm{B}=1.5$ to $2 \mathrm{~T}$, and decreases afterward, showing similar behavior as in the strongly coupled case.

\section{DISCUSSION AND CONCLUSION}

We have computed the Coulomb and exchange energies between two electrons in a double quantum dot system as a function of magnetic fields by using both variational Monte Carlo and exact diagonalization methods. The utilization of simple trial wavefunctions (with $s$ orbitals only) in VMC have lead to good agreement with the ED method, when electrons are decoupled, which validates two-level approaches such as the Heitler-London approximation in weakly coupled QDs. By analyzing the differences between the two methods, we have explicitly demonstrated influence of higher energy single-particle orbitals in the singlet state of the double QDs for strongly coupled systems. We have shown that addition of $p$ orbitals are sufficient enough to accurately describe the system in both the strongly and the weakly coupled QDs. We have also shown that the total Coulomb energies for the singlet and the triplet states behave differently with respect to magnetic field. As indicated by ED and the $s-p$ orbital VMC results, the singlet Coulomb energy in strongly coupled QDs exhibits a minimum in its decrease with magnetic fields because of the strong quenching of the exchange interaction with magnetic localization, whereas this behavior is absent in weakly coupled QDs. The triplet Coulomb energy, however, peaks at intermediate magnetic fields for the same reason, in both strongly coupled and weakly coupled QDs.

\section{ACKNOWLEDGEMENTS}

We are grateful to R. M. Martin and M. Casula for helpful discussion. This work was supported by ARO Grant No. DAAD 19-01-1-0659 under the DARPA-QUIST program and the Material Computational Center. The work performed at the Materials Computation Center was supported by the National Science Foundation under grant no. DMR-03 25939 ITR, with additional support through the Frederick Seitz Materials Research Laboratory (U.S. Dept. of Energy grant no. DEFG02-91ER45439) at the University of Illinois Urbana-Champaign.

(a)

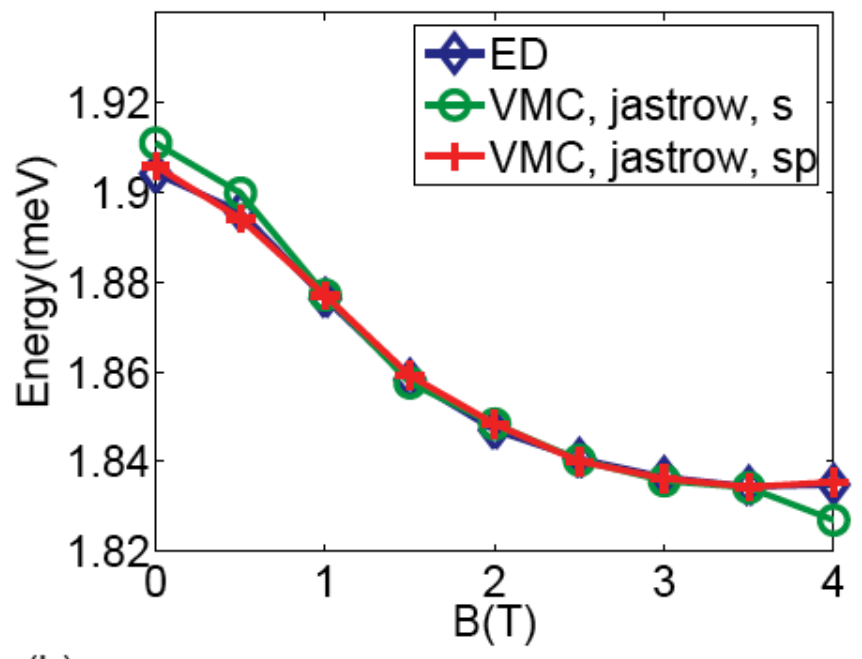

(b)

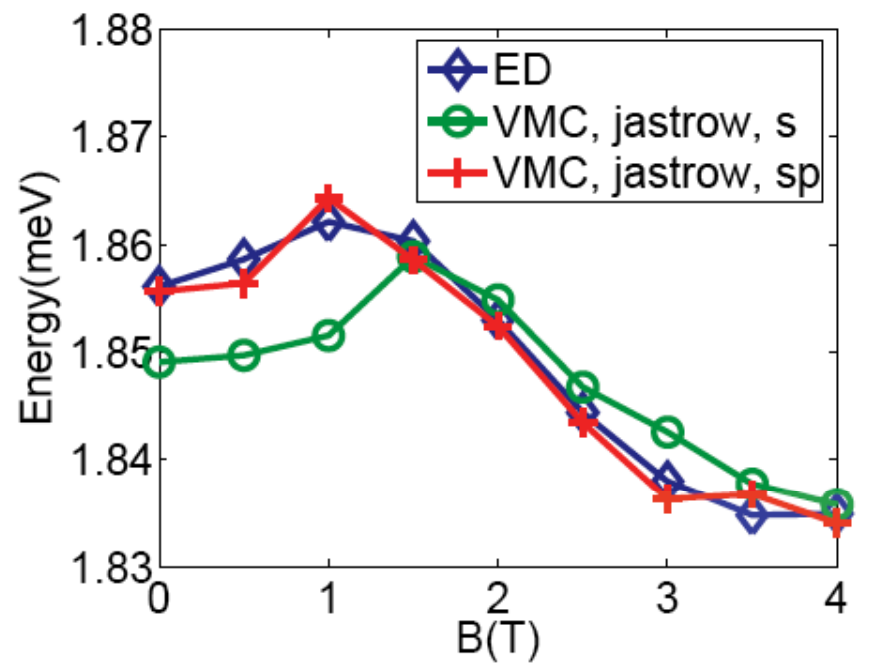

Fig. (4). Total Coulomb energy vs magnetic field in weakly coupled QDs. (a) singlet state and (b) triplet state. 'o'-symbols: VMC ( $s$ orbital); '+'-symbols: VMC ( $s-p$ orbital); ' $\diamond$ '- symbols: ED calculations.

\section{REFERENCES}

[1] Loss D, DiVincenzo DP. Quantum computation with quantum dots. Phys Rev A 1998; 57: 120-6.

[2] Nielsen M, Chuang I. Quantum Computation and Quantum Information. Cambridge Univ. Press: Cambridge; 2000.

[3] Hatano T, Stopa M, Tarucha S. Single-Electron Delocalization in Hybrid Vertical-Lateral Double Quantum Dots. Science 2005; 309(5732): 268-71.

[4] Petta JR, Johnson AC, Taylor JM, et al. Coherent Manipulation of Coupled Electron Spins in Semiconductor Quantum Dots. Science 2005; 309(5744): 2180-4.

[5] McMillan WL. Ground State of Liquid He ${ }^{4}$. Phys Rev 1965; 138 : A442-51.

[6] Ceperley DM, Alder BJ. Monte Carlo simulation of a manyfermion study. Phys Rev B 1977; 16: 3081-99. 
[7] Harju A. Variational Monte Carlo for Interacting Electrons in Quantum Dots. J Low Temperature Phys 2005; 140: 181-210.

[8] Ghosal A, Guclu AD, Umrigar CJ, Ullmo D, Baranger HU. Correlation Induced Inhomogeneity in Circular Quantum Dots. Nat Phys 2006; $2: 336-40$

[9] Saarikoski H, Rasanen E, Siljamaki S, Harju A, Puska MJ, Nieminen RM. Electronic properties of model quantum-dot structures in zero and finite magnetic fields. Euro Phys J B 2002; 26: 241-52.

[10] Melnikov DV, Leburton JP. Single-particle state mixing in twoelectron double quantum dots. Phys Rev B 2006; 73: 155301-5.

[11] Melnikov DV, Leburton JP, Taha A, Sobh N. Coulomb localization and exchange modulation in two-electron coupled quantum dots. Phys Rev B 2006; 74: 041309(R)-12.

[12] Tarucha S, Austing DG, Honda T, van der Hage RJ, Kouwenhoven LP. Shell Filling and Spin Effects in a Few Electron Quantum Dot. Phys Rev Lett 1996; 77(17): 3613-6.
[13] Foulkes WMC, Mitas L, Needs RJ, Rajagopal G. Quantum Monte Carlo simulations of solids. Rev Modern Phys 2001; 73: 33-83.

[14] Kato T. On the eigenfunctions of many-particle systems in quantum mechanics. Commun Pure Appl Math 1957; 10: 151-77.

[15] Fock V, Z Phys. Bemerkung zur Quantelung des harmonischen Oszillators im Magnetfeld. Zeitschrift für Physik A Hadrons and Nuclei 1928; 47: 446-8.

[16] Golovach VN, Loss D. Transport through a double quantum dot in the sequential tunneling and cotunneling regimes. Phys Rev B 2004; 69: 245327-42.

[17] Lin X, Zhang H, Rappe A. Optimization of quantum Monte Carlo wave functions using analytical energy derivatives. J Chem Phys 2000; 112(6): 2650-54.

[18] Burkard G, Seelig G, Loss D. Spin interactions and switching in vertically tunnel-coupled quantum dots. Phys Rev B 2000; 62: 2581-92. 